\title{
Takotsubo Cardiomyopathy a Rare but Serious Complication of Treatment of Primary Central Nervous System Diffuse Large B Cell Lymphoma
}

\author{
Szymanska A1, Taszner M11, Korzon-Burakowska \\ $\mathbf{A}^{\mathbf{1}}$ and Zaucha $\mathrm{J} \mathrm{M}^{1,2 *}$ \\ ${ }^{1}$ Department of Hematology and Transplantology, \\ Medical University of Gdansk, Poland \\ ${ }^{2}$ Department of Hypertension and Diabetology, Medical \\ University of Gdansk, Poland \\ *Correspondling author: ZauchaJ M, Department of \\ Hypertension and Diabetology, Medical University of \\ Gdansk, Poland
}

Received: December 10, 2020; Accepted: February 02, 2021; Published: February 09, 2021

\begin{abstract}
Takotsubo Cardiomyopathy (TC) is a rare, temporary non-ischemic cardiomyopathy usually triggered by emotional stress or mechanical trauma of the brain, however, it might be associated with other Central Nervous System (CNS) diseases. Affected patients present with clinical symptoms of myocardial infarction, cardiac shock that may lead to cardiac arrest. Primary CNS lymphoma is also a rare disease affecting mainly elderly patients, with poor prognosis further aggravated by patient's performance status, comorbidities, early and late treatment related complications. Here we present a 78-year-old patient with primary CNS Iymphoma who unexpectedly developed TC episode following successful chemotherapy treatment.
\end{abstract}

\section{Introduction}

Takotsubo Cardiomyopathy (TC) is a rare temporary nonischemic cardiomyopathy characterized by a temporary wall motion abnormality of the LV and shares common features with Acute Coronary Syndrome (ACS) specifically in terms of a microvascular ACS form with a comparable in-hospital mortality with ST-Segment Elevation Myocardial Infarction (STEMI) and non-STEMI. It is usually triggered by emotional stress or mechanical trauma of the brain. Nowadays it becomes the final diagnosis in about $2 \%$ of all and 5-6 \% of females presenting with suspected ACS however it still might be underdiagnosed [1]. Primary Central Nervous System Lymphoma (PCNSL) is an aggressive form of Non-Hodgkin's Lymphoma (NHL). The disease accounts for $4-5 \%$ of all intracranial brain tumors [2]. It affects mainly patients aged 60-65 years and similarly to other extra nodal lymphomas its incidence $(0,43$ per 100000$)$ is constantly raising [3]. The coincidence of PCNSL and TC has not been described as yet, however CNS tumors and their treatments have been reported as potential triggers of TC [4]. Here we would like to present a patient treated for PCNSL who developed overt TC.

\section{Case Presentation}

A 78-years old women, actively working as medical professional, was admitted to the Department of Hematology and Transplantology at the University Clinical Center in Gdansk in November 2015 with a diagnosis of PCNSL. She presented in July 2015 with increasing headaches, partial vision impairment and a numbness in the upper left limb. Her co-existing diseases were: well controlled hypertension, often migraine headaches and depression treated successfully with anti-depressants. Magnetic resonance imaging revealed a multifocal infiltration of both frontal and right temporal lobe of the brain. A stereotactic biopsy showed the diffuse large B cell lymphoma [LCA (+), CD $20(+)$, bcl6 (+), bcl2 (+/-), CD3 (-), CD10 (-), CD5 (-), Ki67 $(+)$ in $80 \%$ of cells)]. A flow cytometry of spinal fluid showed the presence of $1 \%$ of clonal cells. In summary PCNSL with intermediate risk group categorization according to the MSKCC Prognostic Model
[5], with 4 risk factors according to the International Extranodal Lymphoma Study Group (increased LDH activity, age, elevated level of protein in CS fluid and involvement of deep brain structures in imaging) [6] was diagnosed. At the admission, the patient had no general symptoms, nor consciousness impairments. Before chemotherapy administration, a routine heart function assessment by echocardiography did not reveal any important abnormalities. Patient was offered a reduced MATRix chemotherapy (rituximab, thiotepa $30 \mathrm{mg} / \mathrm{m}^{2}$, methotrexate $3,0 \mathrm{~g} / \mathrm{m}^{2}$ without cytarabine) [7]. The tolerance of chemotherapy was relatively good, elimination of methotrexate was completed within 24 hours without decrease of GFR. The patient improved and was discharged home. The second cycle (with cytarabine) was administered on time (December 2015/January 2016). After achieving a partial response in February 2016, a third cycle was given without any complications. Two days after discharge the patient at home developed sudden consciousness impairmentbecame confused, slept a lot and developed motoric aphasia. The physical examination suggested cardio-pulmonary insufficiency (variable and unstable ranges of blood pressure and crackles on all lung surface). The chest X-ray confirmed massive congestion of lungs; whereas echocardiography showed acute cardiac insufficiency with LVEF drop to $30 \%$ and signs of akinesis and ballooning of apical part of left ventricular consistent with TC [8]. Symptomatic treatment was started and the patient was transferred to the Cardiology Department where after few days of intensive supportive treatment her clinical state improved and she was discharged home. Interestingly, two days later her LVEF improved to $45 \%$ and no previously described changes were present. Chemotherapy was discontinued due to severe treatment related complications and the patient was offered radiotherapy consolidation (36Gy in 18 fractions) with boost to the primary involved fields. The treatment was completed without complications on $8^{\text {th }}$ of May 2016. Few days later the patient was admitted to the emergency department for cardiac arrest that was preceded by the sudden loss of consciousness and seizures. The family started basic life support actions which after 90 seconds led to the restoration of spontaneous heartbeat and breathing. At the admission
Ann Hematol Oncol - Volume 8 Issue 1 - 2021

ISSN : 2375-7965 | www.austinpublishinggroup.com

Zaucha et al. (C) All rights are reserved
Citation: Szymanska A, Taszner M, Korzon-Burakowska A and Zaucha JM. Takotsubo Cardiomyopathy a Rare but Serious Complication of Treatment of Primary Central Nervous System Diffuse Large B Cell Lymphoma. Ann Hematol Oncol. 2021; 8(1): 1324. 
she was still unconscious, Glasgow Scale was 3 points, however her breathing was insufficient and she was transferred to the Intensive care unit to support her breathing. In additional tests there were no signs of acute cardiac insufficiency (LVEF was 55\%), there were no evidence of any CNS abnormalities, severe infection nor pulmonary embolism. After a few hours the patient's breath improved enough to finish active breathing support. During next few days she was recovering quickly and was discharged without neurological or mental symptoms. Since then the follow-up is uneventful and the patient remains in complete response for 4 years coming back to her professional medical duties.

\section{Discussion}

Our case indicates the possibility of coincidence of two exceptional rare phenomena: TC with PCNSL. Our case presented with the most common symptoms of TS that include acute chest pain, dyspnea, or syncope meeting all Inter TAK Diagnostic Criteria of TC from 2018 (except from ECG changes) during the first episode (right after chemotherapy) and only some during the second one [8]. Both episodes were triggered by the same situations: chemotherapy or radiotherapy together with an emotional stress related to it. Importantly, during the first episode the typical, transient changes in the heart wall motions were documented together with slight elevation of cardiac biomarkers (troponin and CPK) that were not observed for the second time. In both situations no ECG abnormalities were documented but all other putative causes such as infection, disease progression, intracranial hemorrhage, pericarditis, myocarditis, and pulmonary embolism were excluded. The etiopathogenesis of TS is unknown. The most likely theory refers to the presumed coronary microcirculation impairment and direct cytotoxicity of high peak endogenous catecholamines induced by extreme stress. There is a long list of predispositions and risk factors that make patients prone to the development of TC such as hormonal factors (predominance of females-89, 9\%, especially at post-menopausal age), genetic factors (variants of receptors) or psychiatric or neurologic disorders TS [9]. In one study, half of the reported patients was suffering from depression [10-12]. Factors directly triggering TC may be emotional or physical. Emotional ones include: grief (e.g. death of a loved one), interpersonal conflicts (e.g. divorce), fear for life (in our case PCNSL with poor prognosis with a median survival of 3 , 5 months [13]) and panic (e.g. robbery or public speaking), anger, anxiety (e.g. personal illness), financial or employment defeat in a competitive (also positive emotions can provoke TC). In our case Physical triggers may be related to medical conditions, or procedures such as acute respiratory failure, traumatic injury, sepsis, malignancy also including chemotherapy and radiotherapy, CNS conditions (e.g. stroke, head trauma, migraine, intracerebral hemorrhage, or seizures as in our case $[1,8,11]$. We cannot exclude that the disease itself (PCNSL) might also contribute to the development of TC affecting the brain integrity and its function. However, it is rather unlikely since TC should have occurred earlier at the time of disease occurrence (as brain tumor) parallelly with first symptoms of CNS involvement or performed biopsy (brain injury). Therefore, the most likely hypothesis is that treatment of PCNSL not PCNSL itself induced TS. The patient received nearly most aggressive treatment at her age with an intention to cure [7]. In the literature there are few papers reporting coincidence of TC with radio/chemotherapy $[14,15]$ but we did not find any report linking PCNSL with TC. Interestingly the second time, the patient suffered from more clinically severe episode of TC, which could be induced by progressive damage of the brain tissue after radiotherapy. Altogether, our patient met several predisposition factors and triggers - she was post-menopausal and suffering from depression and experienced a physical trauma related to the diagnosis of aggressive life threating lymphoma and its treatment. The presented case is so far one of the few that so strongly connects post chemo - and radiotherapy CNS damages with TS. Limitation of our case report is lack of all specific examinations and tests required to be performed during diagnostic work-up. No sequential tests for MRI were taken, no late echocardiography and ECG was performed and finally control coronarography wasn't done to exclude coronary disease. However, the clinical picture of TS and typical performance of echocardiography during the first episode support our diagnosis.

\section{Conclusion}

Takotsubo cardiomyopathy should be taken into differential diagnosis of ACS in patients treated for CNS malignancies.

\section{References}

1. Y-Hassan S, Tornvall P. Epidemiology, pathogenesis, and management of takotsubo syndrome. Clin Auton Res. 2018; 28: 53-65.

2. Nishijima, Daniel K Simel, David L Wisner, David H Holmes, JF PCNSL Update. Curr Onco Res. 2016; 176: 139-148.

3. Han $\mathrm{CH}$, Batchelor TT. Diagnosis and management of primary central nervous system lymphoma. Cancer. 2017; 123: 4314-4324.

4. Finsterer J, Wahbi K. CNS-disease affecting the heart: Brain-heart disorders. J Neurol Sci. 2014; 345: 8-14.

5. Abrey LE. Primary central nervous system lymphoma: The memorial sloankettering cancer center prognostic model. J Clin Oncol. 2006; 24: 5711-5715.

6. Ferreri AJM. Prognostic scoring system for primary CNS lymphomas: The International Extranodal Lymphoma Study Group experience. J Clin Oncol. 2003; 21: 266-272.

7. Ferreri AJM. Chemoimmunotherapy with methotrexate, cytarabine, thiotepa, and rituximab (MATRix regimen) in patients with primary CNS lymphoma: Results of the first randomisation of the International Extranodal Lymphoma Study Group-32 (IELSG32) phase 2 trial. Lancet Haematol. 2016; 3: 217 227

8. Ghadri JR. International Expert Consensus Document on Takotsubo Syndrome (Part I): Clinical Characteristics, Diagnostic Criteria, and Pathophysiology. Eur Heart J. 2018; 39: 2032-2046.

9. Lyon AR. Current state of knowledge on Takotsubo syndrome: A Position Statement from the Taskforce on Takotsubo Syndrome of the Heart Failure Association of the European Society of Cardiology. Eur J Heart Fail. 2016; 18: 8-27.

10. Templin C. Clinical features and outcomes of takotsubo (stress) cardiomyopathy. N Engl J Med. 2015; 373: 929-938.

11. JF, KW. CNS disease triggering Takotsubo stress cardiomyopathy International journal of cardiology. 2014

12. Porto I. Stress cardiomyopathy (tako-tsubo) triggered by nervous system diseases: A systematic review of the reported cases. Int J Cardiol. 2013; 167: 2441-2448.

13. Swerdlow SH. Stress cardiomyopathy (tako-tsubo) triggered by nervous system diseases. Blood J. 2017; 127: 453-462.

14. Desai A, Noor A, Joshi S, Kim AS. Takotsubo cardiomyopathy in cancer patients. 2019

15. Bispo I. Takotsubo syndrome: an overview of pathophysiology, diagnosis and treatment with emphasis on cancer patients. 2019; 833-846. 